Hans-Georg Müller

\title{
Zur textpragmatischen Funktion der Groß- und Kleinschreibung des Deutschen
}

Suggested citation referring to the original publication:

Zeitschrift für germanistische Linguistik 42 (2014), 1, S. 1-25

DOI: http://dx.doi.org/10.1515/zgl-2014-0001

Postprint archived at the Institutional Repository of the Potsdam University in:

Postprints der Universität Potsdam

Philosophische Reihe ; 114

ISSN 1866-8380

http://nbn-resolving.de/urn:nbn:de:kobv:517-opus4-94186 



\title{
Hans-Georg Müller
}

\section{Zur textpragmatischen Funktion der Groß- und Kleinschreibung des Deutschen}

\begin{abstract}
The system of German capitalisation seems to be based on dissimilar levels of linguistic description, i. e. semantics, morphology, and syntax. This leads to several competing scientific models as well as a large range of different rules which appear to be widely autonomous and incompatible with each other. This paper opens an integrative view on the topic by focussing on a pragmatic perspective, which is capable not only on integrating all major application areas of German capitalisation but also of motivating them. Based on the Discourse Representation Theory (DRT) the text pragmatic model can add a functional perspective to established theories by making similar predictions on capitalisation but additionally specifying communicative reasons for them. Therefore it claims explanatory adequacy from the functional perspective.
\end{abstract}

Dr. Hans-Georg Müller: Universität Potsdam, Institut für Germanistik, Am neuen Palais 10, Haus 05, D-14469 Potsdam, E-Mail: hgmuelle@uni-potsdam.de

$\begin{array}{ll}1 & \text { Einleitung } \\ 2 & \text { Diskursrepräsentationstheorie und Majuskelgebrauch } \\ 3 & \text { Das Modell der Diskursinstanzen } \\ 4 & \text { Kernphänomene der Groß- und Kleinschreibung als Diskursinstanzen } \\ 4.1 & \text { Substantive, Substantivierungen und NPn } \\ 4.2 & \text { Ganzsätze, Absätze und Abschnitte } \\ 4.3 & \text { Mehrteilige Eigennamen und „,feste Verbindungen“ } \\ 4.4 & \text { Überschriften und Werktitel } \\ 4.5 & \text { Pronomina und Höflichkeitsanreden } \\ 5 & \text { Ausblick } \\ & \text { Literatur }\end{array}$

\section{Einleitung}

Eine wesentliche textuelle Funktion der deutschen Groß- und Kleinschreibung liegt in der Gliederung des Schriftbildes. Textinitialer und versaler Majuskelgebrauch dienen der Kennzeichnung von Überschriften und Werktiteln, die satzeinleitende Majuskel grenzt maximale syntaktische Strukturen voneinander ab, die satzinterne Groß- und Kleinschreibung erleichtert die Orientierung innerhalb des Textes. 
Nicht abschließend beantwortet ist die Frage, worin die gliedernde Funktion des Majuskelgebrauches eigentlich bestehe. Während ältere theoretische Ansätze semantische und wortkategoriale Merkmale als zentral ansahen, fokussieren jüngere Modelle die Markierung syntaktischer Relationen. Nicht Substantive, sondern Kerne nominaler Gruppen (Maas 1992: 161, Eisenberg 2007: 58) werden großgeschrieben. Dabei scheint ein die Großschreibung bedingendes Kriterium in der Attribuierbarkeit des nominalen Kerns zu liegen. Viele nicht attribuierbare Nominalgruppen waren vor der Rechtschreibreform von der satzinternen Groß- und Kleinschreibung ausgeschlossen. Im Zuge der Neuregelung wurde zwar die syntaktische Perspektive gestärkt, das Kriterium der Attribuierbarkeit hingegen geschwächt, weshalb die amtlichen Regelungen (AR) vielfach kritisiert wurden (vgl. Ickler 1999: 123, 133-134, Munske 2005: 36, Bredel 2010: 224).

Mittlerweile besteht in der Orthografieforschung über die Formel „Großgeschrieben werden attribuierbare Ausdrücke“ (Bredel 2010: 223) weitgehend Einigkeit, während die textlich-funktionalen Ursachen dieser Extension noch weitgehend unerforscht sind. $\mathrm{Zu}$ wenig untersucht wurde bisher die Frage, warum Attribuierbarkeit eine so entscheidende Rolle für nominale Ausdrücke spielt, dass sie als Bedingung der Großschreibung gelten kann. Auch der funktionale Zusammenhang des satzinternen Majuskelgebrauchs mit anderen Anwendungsbereichen der Großschreibung (Überschriften und Werktitel, Ganzsätze, Anredepronomina, mehrteilige Eigennamen) wurde bisher wenig untersucht. In der Diskussion um die Neuregelung der deutschen Rechtschreibung war die Frage nach der textgliedernden Funktion des Majuskelgebrauchs zunächst zugunsten grammatisch-formaler Beschreibungsmöglichkeiten in den Hintergrund getreten.

Der folgende Beitrag möchte einige dieser Lücken schließen und den beschreibungsadäquaten syntaktischen Ansatz durch funktionale Überlegungen ergänzen. Er beabsichtigt, zentrale satzinterne sowie satz- und textinitiale Verwendungsweisen der Majuskel in einem übergreifenden textpragmatischen Modell zu erfassen, das die textgliedernde Funktion der deutschen Groß- und Kleinschreibung spezifiziert und die innere Kohärenz des Gesamtsystems herausstellt. Die Arbeit versteht sich damit als textpragmatisch-funktionales Pendant zur grammatisch-formalen Beschreibung des deutschen Majuskelgebrauchs innerhalb der etablierten orthografischen Modelle. Der gewählte Ansatz ist insofern als textlich zu bezeichnen, als dem Majuskelgebrauch grundlegende textgliedernde und textstrukturierende Aufgaben zugeschrieben werden. Er ist insofern pragmatisch, als diese Aufgaben nicht unabhängig 
von den kommunikativen Absichten des Textemittenten und seiner daraus resultierenden Zeichenverwendung erfüllt werden können. ${ }^{1}$

Das theoretische Fundament bildet die Diskursrepräsentationstheorie (DRT), die sich als Ausgangspunkt anbietet, da sie eine funktionale Perspektive auf den Aufbau von Texten eröffnet und dabei rekursive textliche Strukturebenen annimmt, die auch im Bezug auf den Majuskelgebrauch auszumachen sind (vgl. Abschnitt 3). Durch Majuskel gekennzeichnete Textteile weisen große Ähnlichkeiten zu Diskursreferenten auf und sollen daher in Anlehnung an die DRT als Diskursinstanzen bezeichnet werden.

\section{Diskursrepräsentationstheorie und Majuskelgebrauch}

Die Diskursrepräsentationstheorie (DRT) geht auf die Arbeiten von Kamp (1981) sowie Kamp \& Reyle (1993) zurück und versucht, das grammatische Verhalten von definiten und indefiniten NPn sowie anaphorischen Ausdrücken innerhalb eines Textes zu spezifizieren. Kamp \& Reyle verwenden dazu den von Karttunen (1976) entlehnten Begriff des Diskursreferenten.

Diskursreferenten sind rekursiv aufgebaute und hierarchisch angeordnete Einheiten des Textes. Sie lassen sich als sprachliche Gegenstände betrachten, die im Text diskursiv miteinander in Beziehung gesetzt, über die Aussagen gemacht und deren Eigenschaften zur Disposition gestellt werden. Die Menge aller Diskursreferenten eines Textes bilden das Diskursuniversum, die Menge aller Beziehungen unter ihnen bildet die Diskursrepräsentationsstruktur (DRS, vgl. Kamp \& Reyle 1993: 63).

Für die Groß- und Kleinschreibung ist die DRT deshalb interessant, weil die Menge der Diskursreferenten eines Textes identisch ist mit der Menge großgeschriebener Entitäten. So bilden etwa Eigennamen und echte Substantive stets auch Diskursreferenten, die innerhalb des Diskurses in Beziehung zueinander treten. Diskursreferenten drücken damit in textpragmatischer Begrifflichkeit aus, was Ewald \& Nerius (1990: 35 f) als „gegenständlich im weitesten Sinne“ bezeichnen und zur Grundlage der Substantivgroßschreibung machen. Die Gegenständlichkeit

\footnotetext{
1 Aus Platzgründen ist die Darstellung auf zentrale Anwendungsbereiche der orthografischen Norm beschränkt. Nicht bzw. nur randlich behandelt werden insbesondere Versalschreibungen, Durchkupplungen, Majuskelgebrauch in Akronymen und Abkürzungen sowie die sog. Binnenmajuskel. Eine umfassende theoretische wie empirische Untersuchung befindet sich derzeit kurz vor dem Abschluss. In ihr werden auch didaktische Konsequenzen gezogen, die in diesem Beitrag ebenfalls unberücksichtigt bleiben müssen.
} 
von Diskursreferenten resultiert nicht primär aus semantischen oder morphosyntaktischen, sondern aus diskursiven Eigenschaften, die eng mit grammatischen Merkmalen von Nominalität assoziiert sind, ohne mit ihnen deckungsgleich zu sein. Der Gedanke der Kennzeichnung von Diskursreferenten durch Majuskel kann deshalb den Großteil der gegenwärtigen orthografischen Norm erklären, der in den AR mithilfe von mehrstufigen Grund- und Ausnahmeregeln erfasst wird. So existieren etwa sprachliche Strukturen, die wortkategoriale bzw. syntaktische Eigenschaften von Nominalität aufweisen, aber nicht großgeschrieben werden.

(1) Die ganze Gesellschaft stand kopf.

(2) Ihr könntet euch ein bisschen zusammenreißen.

Obwohl $\mathrm{K} /$ kopf dem lexematisch-paradigmatischen wie dem wortsemantischen Substantivkonzept i.S. Gallmanns (1997: 220 f, 232 f) genügt und ein B/bisschen sowohl morphologische als auch syntaktische Nominalitätskriterien aufweist, werden beide Entitäten unter Inkaufnahme von Sonder- und Ausnahmeregeln von der Großschreibung ausgeschlossen (§ 56 (2), (5) AR). ${ }^{2}$ Ihr besonderer grammatischer Charakter, aus dem die Kleinschreibung resultiert, lässt sich mithilfe syntaktischer Proben ausweisen, liefert damit aber noch keine unmittelbaren Begründungen für ihren Ausschluss von der Großschreibung (vgl. Abschnitt 4.1). Die DRT vermag hier einen funktional begründbaren Anhaltspunkt $\mathrm{zu}$ geben, ab welchem Grad von Nominalität Majuskelgebrauch angebracht ist: So gehört $\mathrm{zu}$ den grammatischen Eigenschaften von Diskursreferenten, dass sie stets durch anaphorische Verweise wiederaufnehmbar und attribuierbar sind. Beide Fähigkeiten stellen keine zufälligen formalen Kriterien dar, sondern resultieren aus der Funktion von Diskursreferenten für den Text (vgl. Abschnitt 3). Mit der DRT kann die Kleinschreibung von (1) und (2) daher nicht nur grammatisch motiviert, sondern zusätzlich funktional begründet werden. Bei beiden handelt es sich, wie zu sehen sein wird, nicht um Diskursreferenten, die sich zur Thematisierung und ggf. späteren Wiederaufnahme im Text eignen. Nur wenn eine textliche Entität ein „Redegegenstand [...] d. h. etwas, wovon die Rede ist, und nicht nur eine modalisierende, quantifizierende, intensivierende oder textorganisierende Floskel“ ist (Ickler 1999: 134), kann sie als relevant für die Groß- und Kleinschreibung betrachtet werden.

Darüber hinaus ermöglicht die DRT eine funktionale Verbindung von satzinterner, satzinitialer und textinitialer Großschreibung (vgl. Abschnitt 4). Nicht nur Substantive, sondern auch Sätze lassen sich als Diskursreferenten beschreiben, die anaphorisch aufgenommen und attribuiert werden können. Sie unter-

2 Beide Entitäten widersprechen damit Gallmanns Grundsatz, dass großgeschrieben werde, was mindestens einem Nominalitätskonzept genüge. 
scheiden sich damit nicht kategorial, sondern lediglich hierarchisch von anderen Diskursreferenten. ${ }^{3}$ Die DRT nimmt dafür rekursive Strukturen an, bei denen Verknüpfungsregeln zwischen Diskursreferenten zu komplexen Einheiten führen, die sich ihrerseits als Diskursreferenten verstehen lassen. Diese Verknüpfungsregeln bilden die Diskursrepräsentationsstruktur und erklären den Aufbau von Texten als die Verknüpfung von Wörtern zu Sätzen, von Sätzen zu Absätzen, zu Kapiteln und schließlich zum Textganzen. Jede der dabei entstehenden Hierarchieebenen wird durch initiale Majuskel ausgezeichnet.

\section{Das Modell der Diskursinstanzen}

Die DRT verfolgt andere als orthografische Ziele. Ihr Fokus liegt nicht auf den Aspekten, die für den hier zu entwickelnden Zusammenhang von Bedeutung sind. ${ }^{4}$ Aus diesem Grund soll in der Folge der Begriff der Diskursreferenten vermieden und stattdessen von Diskursinstanzen gesprochen werden. Diskursinstanzen sind Informationseinheiten des Textes, auf die, ähnlich wie auf Diskursreferenten, im weiteren Verlauf des Textes anaphorisch Bezug genommen werden kann. Darüber hinaus kommt Diskursinstanzen das Kriterium der Attribuierbarkeit zu, wie im Folgenden hergleitet wird. Die Menge der attribuierbaren Ausdrücke des Textes kongruiert mit der Menge seiner Diskursinstanzen.

Als Instanz einer abstrakten Klasse $X$ bezeichnet man die konkrete Ausprägung $x$ eines Objektes dieser Klasse mit charakteristischen, für $x$ spezifizierten Parametern. So ist etwa Dackel eine mögliche Instanz der Klasse Hund mit spezifischen Parametern für Größe und Aussehen, die ihn von anderen Instanzen der Klasse Hund unterscheiden. Bruno, der Hund meines Nachbarn, ist wiederum eine Instanz der Klasse Dackel, der in allen relevanten Parametern als Instanz dieser Klasse ausgewiesen ist, darüber hinaus aber noch bezüglich der Parameter Alter, Geschlecht, Gesundheitszustand etc. weiter spezifiziert ist. Die Ableitung eines Objektes $x$ aus einer abstrakten Klasse $X$ wird als Instantiierung bezeichnet.

Diskursinstanzen lassen sich damit als konkrete Realisationen abstrakter Klassen begreifen, mit denen innerhalb des Diskursuniversums auf tatsächliche oder denkbare Gegenstände und Sachverhalte referiert wird. Aus dieser Eigen-

3 In der DRT werden auch (Teil-)Sätze unter bestimmten Umständen als eigene Diskursreferenten betrachtet (vgl. Kamp \& Reyle 1993: 483-487), wenngleich aus hier zunächst nicht relevanten Gründen.

4 Die DRT geht insbesondere der Frage nach, unter welchen Bedingungen phorische Verweise auf Diskursinstanzen innerhalb von abhängigen Gliedsätzen möglich sind, während der Verweis auf Sätze selbst eine untergeordnete Rolle spielt. 
schaft resultiert ihre Potenz, durch Pronomina anaphorisch wiederaufnehmbar zu sein, die als erstes Operationalisierungskriterium zur Bestimmung von Diskursinstanzen herausgestellt werden kann.

Während der Instantiierung einer Diskursinstanz $x$ aus einer abstrakten Klassen $X$ kommt es zur Spezifizierung konkreter Parameter, die $x$ als Attribute ${ }^{5}$ zukommen und sie von anderen Instanzen der Klasse $X$ unterscheiden. Tatsächlich dienen Diskurse vorrangig dazu, die Attribute von Diskursinstanzen darzustellen, zu ändern, zu erfragen oder anderweitig zur Disposition zu stellen. Für Diskursinstanzen unterhalb der Satzebene, die in der Regel (aber nicht immer) aus semantisch-konzeptuellen Klassen abgeleitet werden, kann der Attributbegriff dabei durchaus grammatisch verstanden werden, wenngleich nicht jedes Attribut einer Diskursinstanz auch grammatisch als Attribut realisiert werden muss. Entscheidend ist die Potenz jeder Diskursinstanz, bezüglich bestimmter Eigenschaften spezifiziert zu sein. Aus dieser Potenz leitet sich das zweite Operationalisierungskriterium, die (grammatische) Attribuierbarkeit, ab, die bereits im syntaktischen Ansatz als konstitutiv für die satzinterne Groß- und Kleinschreibung herausgestellt wurde.

Beide Operationalisierungskriterien - pronominale Referenzierbarkeit wie Attribuierbarkeit - erweisen sich bei genauer Betrachtung als zu mächtig und sind in Einzelfällen auch auf Nicht-Diskursinstanzen anwendbar. Beispielsweise sind pronominale Ausdrücke häufig rechtsattribuierbar, obwohl sie selbst keine Diskursinstanzen sind, sondern lediglich auf solche verweisen. Ferner sind auch syntaktische Phrasen zum Teil anaphorisch wiederaufnehmbar und, einen weiten Attributbegriff vorausgesetzt, auch attribuierbar. Diesem Problem, das auch dem syntaktischen Ansatz der Groß- und Kleinschreibung eigen ist, kann einerseits durch zusätzliche Beschränkung der Operationalisierungskriterien begegnet werden. So lässt sich etwa Attribuierbarkeit auf Linksattribution mit flektierten Adjektiven beschränken (vgl. etwa Röber-Siekmeyer 1999: 64 f, 110), pronominale Referenzierbarkeit auf Wiederaufnehmbarkeit mit anaphorischen Personalpronomina. Diese Beschränkungen grenzen den Problemkreis stark ein, bilden aber nur einen oberflächlichen Ausweg. Das eigentliche Problem liegt darin, dass Referenzierbarkeit und Attribuierbarkeit zwar schlüssig aus der Definition von Diskursinstanzen ableitbar sind, dass diese Beziehung jedoch nicht vollständig bikonditional ist. Die Operationalsierungskriterien sind hinreichend trennscharf, um sinnvolle Bestimmungen von Diskursinstanzen zu ermöglichen, sie ersetzen

5 Der dabei zugrundegelegte Attributbegriff geht hier über den grammatischen hinaus und folgt informationstheoretischen Überlegungen. 
aber nicht das Verständnis ihrer diskursiven Funktion. In den Einzeluntersuchungen wird auf diesen Punkt zurückzukommen sein.

Diskursinstanzen bilden konsequent Hierarchieebenen, welche als rekursiv zu bezeichnen sind, da die Verknüpfung von Diskursinstanzen der Ebene $n_{O} \mathrm{zu}$ Strukturen führt, die auf der Ebene $n_{1}$ ihrerseits als Diskursinstanzen bezeichnet werden können. Für jede Hierarchieebene haben sich charakteristische Formen der Referenzierung und der Attribution sowie korrespondierende graphemische Markierungskonventionen herausgebildet, so etwa Satzschlusszeichen, Absatzmarkierungen, Abschnitts- und Seitenwechsel. Allen Ebenen ist gemein, dass ihre Entitäten durch eine Majuskel initialisiert werden. ${ }^{6}$ Da in vielen Anwendungsfällen nur im Hinblick auf den textlichen Zusammenhang darüber entschieden werden kann, was als Diskursinstanz fungiert und was nicht, ist die Domäne der Diskursinstanz der Text.

\section{Kernphänomene der Groß- und Kleinschreibung als Diskursinstanzen}

\subsection{Substantive, Substantivierungen und NPn}

Viele traditionelle Modelle zur Erklärung der satzinternen Groß- und Kleinschreibung gingen von einem grundlegend semantisch definierten Substantivbegriff als dem zentralen Gegenstand des Majuskelgebrauchs aus. So versuchen Ewald \& Nerius (1990), das Konzept der Gegenständlichkeit konkreter Appellativa auf abstrakte Substantivklassen zu übertragen (,gegenständlich im weitesten Sinne“, s. o.). Dieser Versuch reicht hin, um eine intuitiv verständliche Gemeinsamkeit aller Substantive anzudeuten, ist aber definitorisch begrenzt, da sich über semantische Merkmale keine operationalisierbaren Kriterien des Majuskelgebrauchs ableiten lassen. Deshalb wird der Substantivbegriff sowohl bei Ewald \& Nerius (1990: 34-35) als auch in den AR zusätzlich durch morphologische und syntaktische Kriterien wie Numerus- und Genusspezifikation, Artikelfähigkeit und die Einnahme bestimmter Satzgliedpositionen präzisiert (vgl. §§ 55, 57 AR). Aus dieser Operationalisierung resultieren Abgrenzungsprobleme, die eine breite und schwer plausibel normierbare schriftsprachliche Peripherie verursachten.

6 Auf Phänomene des mehrfachen Majuskelgebrauchs innerhalb einer Diskursinstanz kann aus Platzgründen nur randlich eingegangen werden, vgl. Abschnitt 4.3. 
Die Probleme des Substantivbegriffs verlieren sich, wenn man Gegenständlichkeit textpragmatisch auffasst, wie es das Modell der Diskursinstanzen erlaubt. Unabhängig von ihren semantischen Eigenschaften lassen sich Substantive allgemein als diskursive Gegenstände auffassen, die im Text in propositionale Zusammenhänge zueinander gestellt werden. Gegenständlichkeit im diskursiven Sinne, und damit der Charakter einer schriftsprachlichen Entität als Diskursinstanz, manifestiert sich in ihrer Eigenschaft, im Beziehungsgeflecht des Diskursuniversums eine spezifische Position zu besetzten und dort diskursiv „ansprechbar“ zu sein. Aus dieser Eigenschaft resultiert neben der Attribuierbarkeit die Fähigkeit zur anaphorischen Wiederaufnahme im Folgetext. ${ }^{7}$ Auf Satz (3) lassen sich genau die durch Majuskel ausgezeichneten Wörter zum anaphorischen Anschluss nutzen, wie (4) bis (7) zeigen.

(3) Fritz hatte keine Angst vor dem Dackel der Nachbarin, denn...

(4) ... er war schließlich kein Angsthase.

(5) ... die hatte er bereits vor Jahren abgelegt.

(6) ... der war schließlich alt und grau.

(7) ... sie hatte ihren Bruno gut erzogen.

Der Eigenname Fritz und die Konkreta Dackel und Nachbarin verhalten sich textlich gesehen nicht anders als das Abstraktum Angst. Eine Unterscheidung unterschiedlicher Substantivklassen ist nicht erforderlich.

Aus der textpragmatischen Perspektive auf die Substantivgroßschreibung ergeben sich unmittelbare Konsequenzen für einige in der Forschung diskutierte Problemkreise, namentlich zu Substantivierungen und Desubstantivierungen sowie zur Schreibung nichtattribuierbarer nominaler Phrasen. Substantivierungen lassen sich diskursiv als Operationen betrachten, um beliebige Texteinheiten $\mathrm{zu}$ instantiieren und damit zu Gegenständen des Diskurses zu machen. Der Unterschied zwischen (8) und (9), der sich im unterschiedlichen Majuskelgebrauch niederschlägt, liegt diskursiv darin, dass sich der durch das Morphem [alt] angesprochene Sachverhalt in (9) unmittelbar anaphorisch wiederaufnehmen und attribuieren lässt, in (8) hingegen nicht. Will man entsprechende diskursive Eigenschaften auch bei (8) herstellen, muss man zuvor Instantiierungsoperationen durchführen, etwa durch Derivationsprozesse.

(8) Der Dackel ist schon sehr alt. ${ }^{*}$ Es beträgt fast 16 Jahre.

(9) Der Dackel hat ein hohes Alter. Es beträgt fast 16 Jahre.

7 Ausnahmen finden sich bei Konstruktionen, die auch in anderen theoretischen Entwürfen problematisch sind - so etwa bei Funktionsverbgefügen, s. u. 
Aus textpragmatischer Perspektive liegt der entscheidende Punkt der Substantivierung nicht in der Anwendung eines morphologischen Bildungsverfahrens (alt => Alter), sondern im diskursiven, von der Intention des Textproduzenten abhängigen Bedürfnis zur Instantiierung eines mentalen Konzepts, das anschließend als „Gegenstand“ dem weiteren Diskursverlaufs zur Verfügung steht. Das zeigt sich auch bei spontanen Substantivierungen wie in (10), denen keine derivationalen Bildungsmuster anzusehen sind. Die Potenz zur anaphorischen Wiederaufnahme weist Sitz unabhängig von seinem kategorialen Status als Diskursinstanz aus.

(10) Der Hund hat dein „Sitz“ wohl nicht gehört. Du wirst es wiederholen müssen.

Analog lassen sich Desubstantivierungen betrachten. Der Unterschied zwischen Substantiven und Desubstantivierungen liegt nicht, wie $\S 56$ AR formuliert, darin, dass Desubstantivierungen „selbst keine substantivischen Merkmale aufweisen“ (damit widersprächen die AR ihrer eigenen semantischen Substantivdefinition) sondern darin, dass die entsprechenden textlichen Entitäten keine Diskursinstanzen (mehr) sind. Die Kleinschreibung von (11) gegenüber (12) ist einfacher diskursiv als über das Vorhandensein bzw. Fehlen substantivischer Merkmale zu motivieren. ${ }^{8}$

(11) Petra stand kopf. ${ }^{\star}$ Er schmerzte ihr hinterher.

(12) Petra stand auf dem Kopf. Er schmerzte ihr hinterher.

In der jüngeren Forschung ist die wortkategoriale Perspektive zunehmend durch den syntaktischen Ansatz ersetzt worden, was sich auch in den AR niedergeschlagen hat. Dabei konnte gezeigt werden, dass Nominalität keine kategoriale Eigenschaft ist, sondern sich vielfach aus dem syntaktischen Kontext ergibt (vgl. Fuhrhop 2007: 5). Über den syntaktischen Ansatz lassen sich nicht nur Probleme der Substantivierung und Desubstantivierung auf elegante Weise lösen, sondern auch etablierte Proben (etwa die Artikelprobe) plausibel begründen. Fuhrhop (2007: 32 ff) stellt eine Reihe von Tests zusammen, mit denen der nominale Charakter schriftsprachlicher Entitäten geprüft werden kann. Mithilfe dieser Tests, darunter neben Artikel- und Attribuierungsprobe auch die Negationsprobe mit „nicht“ oder „kein“, die Verwendbarkeit der Entität als Objekt sowie verschiedene topologische Prüfmethoden, kann überzeugend gezeigt werden, dass K/kopf in (11) und (12) unterschiedliche Grade von Nominalität aufweist. Nicht direkt erschließbar ist hingegen, ab welchem Grad Großschreibung erfolgen sollte, weshalb gerade das Beispiel K/kopf stehen in der Normdiskussion stark umstritten war. Vor der Rechtschreibre-

8 Das ist freilich auch möglich, liefert aber lediglich grammatische Befunde, deren Bedeutung für den Majuskelgebrauch strittig sein kann. Die diskursive Sicht bietet demgegenüber eine funktionale Erklärung, die mit den grammatischen Eigenschaften korrespondiert (s.u.). 
form war die Entität kleingeschrieben worden, in der Fassung von 1996 sahen die AR Großschreibung vor, mit der Revision von 2006 wieder Kleinschreibung.

Zurecht weist Fuhrhop (2007) darauf hin, dass syntaktische Tests zum Nachweis von Nominalität als „Hilfsmittel, sprachliches Wissen abzuprüfen“ (ebd. 32) verstanden werden müssen. Dieses sprachliche Wissen erschöpft sich nicht in formalen Aspekten, sondern kann auch funktional gedeutet werden. Grammatische Operationen wie Determination oder Attribution sind nicht funktionslos, sondern dienen bestimmten diskursiven Zwecken. Beispielsweise spezifiziert Determination die referenziellen Eigenschaften einer Diskursinstanz, Attribution expliziert die außersprachlichen Eigenschaften des Referenzobjektes. Fehlen die diskursiven Zwecke, etwa weil Referenz auf einen außersprachlichen Gegenstand gar nicht intendiert ist, werden auch die entsprechenden grammatischen Operationen obsolet. ${ }^{9}$ In (11) ist im Gegensatz zu (12) weder Referenz noch eine Instantiierung, d.h. eine Spezifizierung konkreter Eigenschaften des abstrakten semantischen Konzepts beabsichtigt. Die besonderen grammatischen Eigenschaften von (11) korrespondieren mit der spezifischen Funktion der schriftsprachlichen Entität für den textlichen Diskurs.

Die jüngste Forschung hat sich mit dem Kriterium der Attribuierbarkeit auf eine syntaktisch prüfbare nominale Eigenschaft geeinigt, die als Bedingung des Majuskelgebrauchs gilt. Dennoch wurde Attribuierbarkeit als Kriterium nicht in die amtliche Norm aufgenommen, ${ }^{10}$ da sie syntaktisch nur eines von mehreren nominalen Prüfverfahren zu sein schien, dessen außerordentliche Bedeutung für die Groß- und Kleinschreibung zwar aus der tradierten orthografischen Konvention abgeleitet werden konnte, aber innerhalb des orthografischen Systems entbehrlich schien. Diskursiv lässt sich die Bedeutung der Attribuierbarkeit für den Majuskelgebrauch aus dem Prozess der Instantiierung abstrakter Klassen herleiten. Das Modell erweist sich als zu hundert Prozent vereinbar mit Bredels Postulat „Großgeschrieben werden attribuierbare Ausdrücke“ (s. o.) und kann die Gültigkeit dieses Grundsatzes zusätzlich textpragmatisch begründen. Nach Maßgabe des Instanzenmodells ist Attribuierbarkeit eine notwendige Folge der Ableitung einer Diskursinstanz aus einer abstrakten Klasse.

9 Die dazugehörigen diachronen Veränderungsprozesse haben Bredel \& Günther (2000: 106109) in einem Kontinuum verblassender Nominalität beschrieben, mit dem sich schrittweise die grammatischen Eigenschaften nominaler Phrasen ändern.

10 Vgl. insbesondere $§ 55$ (4) und 57 (1), sowie die Diskussion der Paragrafen bei Gallmann \& Sitta 2007: 29 ff. 
Dass die diskursive Perspektive hierbei die ursächliche ist, aus welcher die (Nicht-) Attribuierbarkeit folgt, zeigt sich daran, dass sich Kontexte konstruieren lassen, in denen sonst stereotypisierte Phrasen, die nach den alten Regeln von der Großschreibung ausgeschlossen waren, in wörtlicher Bedeutung verwendet und dabei zu Diskursinstanzen werden: ${ }^{11}$

(13) Fritz tappte im schwärzesten Dunkeln, das ihn je umgeben hatte.

„Das Dunkle“ wird in (13) nicht in der übertragenen Bedeutung, sondern als Diskursinstanz verwendet, ist daher unmittelbar attribuierbar und kann anaphorisch wiederaufgenommen werden. Die Attribuierbarkeit einer Phrase ergibt sich nicht aus ihren syntaktischen Eigenschaften, sondern aus ihrer diskursiven Verwendung. ${ }^{12}$

Dass der Beispielkontext in (13) künstlich wirkt, ist kein Zufall, sondern hängt mit den pragmatischen Eigenschaften stereotypisierter Phrasen zusammen, von denen auch ihre Nicht-Attribuierbarkeit folgt. Stereotypisierte Phrasen dienen dazu, kommunikative Standardsituationen ohne größeren diskursiven Aufwand zu bewältigen. (14) Ich geh im A/allgemeinen dreimal täglich mit dem Hund raus.

Wer eine Phrase wie (14) äußert, möchte gerade keinen Diskurs darüber eröffnen, was unter „dem Allgemeinen“ zu verstehen sei. Stereotypisierte Phrasen lassen sich als kommunikativen Versuch deuten, die diskursive Behandlung des entsprechenden Phraseninhaltes zu unterbinden und den Fokus auf andere Bereiche der Äußerung zu lenken. Das „Allgemeine“ ist keine Konkretisierung einer abstrakten Klasse mithilfe spezifischer Eigenschaften. Es lässt sich folglich nicht als Instanz des Textes auffassen, weshalb auch Attribuierung nicht möglich ist. Sie würde der kommunikativen Funktion der Phrase widersprechen.

Das Instanzenmodell bildet eine praktikable Möglichkeit, den Bereich der sog. Substantivgroßschreibung so zu umreißen, dass alle unstrittigen Phänomene des Majuskelgebrauchs inbegriffen sind, während zu den umstrittenen dezidiert Stellung genommen wird. Typische Prüfverfahren, insbesondere das Kriterium der Attribuierbarkeit, werden integriert und sind in ihrer Bedeutung für die Groß-

11 Dieser Umstand erklärt auch die semantische - eigentlich pragmatische - Unterscheidung der alten Regeln, ob feststehende Wendungen wie „im Dunkeln tappen“ im wörtlichen oder übertragenen Sinne gemeint sind, vgl. Gallmann \& Sitta 2007: 31. Nur im wörtlichen Sinne ist Attribution wie in (13) möglich, weil nur hier eine Diskursinstanz vorliegt.

12 Es ist in diesem Zusammenhang wichtig zu unterscheiden, ob man die syntaktischen Eigenschaften einer schriftsprachlichen Entität untersucht oder ob man einen syntaktischen Test anwendet, um sich die pragmatischen Eigenschaften der Entität zu vergegenwärtigen. Mit dem Test auf Attribuierbarkeit einer Phrase tut man letzteres (vgl. Fuhrhop 2007: 32). 
und Kleinschreibung erklärbar. Substantivierung und Desubstantivierung lassen sich unschwer als Funktionen der diskursiven Struktur von Texten einbinden.

\subsection{Ganzsätze, Absätze und Abschnitte}

Satzinitialer und satzinterner Majuskelgebrauch werden in gängigen orthografischen Modellen in der Regel als getrennte Regelkomplexe behandelt, obwohl die graphemische Gemeinsamkeit der Initialisierung durch Majuskel sicherlich kein Zufall ist. ${ }^{13}$ Durch Herausstellung der textpragmatisch-funktionalen Verwandtschaft beider Phänomene kann diese Trennung abgebaut werden. Für den Text sind Wörter, Sätze oder Absätze in erster Linie Informationseinheiten unterschiedlicher Komplexität. In dieser Gemeinsamkeit liegt eine mögliche Ursache für den gemeinsamen Majuskelgebrauch, der mit ähnlichen diskursiven Eigenschaften einhergeht und damit auch die Anwendung ähnlicher Proben zulässt.

Der auf Glinz (1973: 26) zurückgehende Ganzsatzbegriff ist oft als tautologisch abgelehnt worden (vgl. etwa Ickler 1998: 117, 125), hat sich aber dennoch in den amtlichen Regelungen halten können, was nicht zuletzt an seiner Alltagstauglichkeit für die Schreibpraxis sowie am Mangel präziserer und dennoch didaktisch verwertbarer Alternativen liegt. So schwer es ist, den Ganzsatz-Begriff grammatisch zu fassen (vgl. etwa Ewald \& Nerius 1990: 26, Glinz 1984: 43-44), so leicht fällt es geübten Schreibern, sinnvoll mit satzinitialer Majuskel und Satzschlusszeichen umzugehen. Das dürfte neben prosodischen Ursachen auch daran liegen, dass die Domäne des Ganzsatzes nicht die Syntax sondern die Textpragmatik ist.

Bereits Stetter (1990: 302) definiert den Ganzsatz konsequent pragmatisch: Seiner Meinung nach sind Texte nicht aus Sätzen, sondern aus illokutiven Schreibakten aufgebaut. Die Grenzen dieser Schreibakte definiert der Emittent durch Majuskelgebrauch und Satzzeichen. Stetters Ansatz weiter verfolgend können Ganzsätze in das textpragmatische Modell des Majuskelgebrauchs integriert werden, da sie in wichtigen Eigenschaften Gemeinsamkeiten zu bisher dargestellten Diskursinstanzen aufweisen und durch die gleichen Operationalisierungskriterien identifizierbar sind. Auch Ganzsätze lassen sich attributiv er-

13 Sowohl zeichentheoretisch als auch historisch lassen sich Argumente finden, die eine funktionale Unabhängigkeit unwahrscheinlich machen. Echte Homonymie ist wesentlich seltener als Polysemie. 
weitern $(15)^{14}$ und auch sie verfügen über die Eigenschaft, anaphorisch wiederaufnehmbar zu sein (16). ${ }^{15}$

(15) Fritz war schon mit dem Hund draußen, was nicht alle Tage vorkommt.

(16) Fritz war schon mit dem Hund draußen. Das hat mich sehr verblüfft.

Beide Prüfmechanismen dürfen nicht als Ursache, sondern müssen als Indikator aufgefasst werden, dass es sich bei Ganzsätzen um Arten von Diskursinstanzen handelt. Jeder illokutive Schreibakt wird durch seine Stellung im Diskursuniversum zum konstitutiven Gegenstand des Textes, auf den textintern- wie textextern Bezug genommen wird und dem Eigenschaften zugeschrieben werden können. Wie satzinterne Diskursinstanzen sind auch Ganzsätze diskursiv „ansprechbar“, besetzen also eine spezifische Position innerhalb der DRS und treten in Beziehung zu anderen Instanzen der gleichen Hierarchieebene. Zwischen satzinternen Diskursinstanzen ist diese Beziehung propositional, zwischen Instanzen der Satzund Absatzebene diskursstrukturell und folgt damit anderen, aber funktional vergleichbaren Verknüpfungsregeln. Die diskursiven Beziehungen, in denen Instanzen gleicher Ebene zueinander stehen, charakterisieren die interne Struktur der aus ihnen konstituierten Diskursinstanz nächsthöherer Hierarchiestufe.

Durch rekursiv-hierarchische Anwendung des Instanzenmodells lassen sich textliche Makrostrukturen wie Absätze, Abschnitte und Kapitel ebenfalls als Dis-

14 Der Attributbegriff ist auf (15) insofern anwendbar, als der weiterführende Nebensatz eine diskursstrukturelle Beifügung zur vorausgegangenen Proposition ist, vgl. auch Anm. 5.

15 Ein anonymer Gutachter weist darauf hin, dass sich unter bestimmten Umständen nicht nur Ganzsätze, sondern auch abhängige Nebensätze anaphorisch wiederaufnehmen lassen, so etwa Objektnebensätze.

Fritz hoffte, dass der Dackel gegen Tollwut geimpft ist. Er wusste das aber nicht genau.

Wiederaufnahmemöglichkeiten von Nebensätzen sind systematisch möglich, jedoch häufig das Resultat plausibler kontextspezifischer Interpretationen. Fällt der diskursive Kontext uneindeutig aus, wird auch der anaphorische Verweis ambig.

Fritz hoffte, dass der Dackel gegen Tollwut geimpft ist. Seine Mutter wusste das.

Die Beispiele belegen in erster Linie, dass anaphorische Referenzierung nicht losgelöst vom diskursiven Kontext betrachtet werden kann. Obwohl der Bezug auf den Ganzsatz der Standardfall der diskursiven Interpretation sein dürfte und ein Großteil der Nebensatzkonstruktionen nicht von außen anaphorisch referenzierbar ist, liegt das eigentliche Problem darin, dass das Verhältnis von Diskursinstanzen und Operationalisierungskriterien nicht vollständig bikonditional ist. Dieser Umstand wurde bereits in Abschnitt 3 angesprochen. Er besagt, dass das Phänomen Diskursinstanz nicht mit seinen Operationalisierungskriterien verwechselt werden darf. 
kursinstanzen höherer Ordnung ansehen. ${ }^{16}$ Bei Hierarchieebenen oberhalb des Ganzsatzes, wie Absätzen, Abschnitten und Kapiteln ist zu beachten, dass sich ihr Charakter als Diskursinstanz nicht ohne weiteres durch die oben entwickelten Proben operationalisieren lässt. Das liegt daran, dass sich für Absätze, Abschnitte und Kapitel keine grammatikalisierten Prozeduren der Attribuierung oder anaphorischen Wiederaufnahme entwickelt haben. Eine andere Möglichkeit der operationalen Integration hängt eng mit dem Problem der Überschriften und Werktitel zusammen und wird deshalb an anderer Stelle diskutiert (vgl. Abschnitt 4.4).

\subsection{Mehrteilige Eigennamen und „feste Verbindungen“}

Eigennamen bilden den Ursprung und den ältesten Bereich des satzinternen Majuskelgebrauchs. Historisch gesehen hat sich die Substantivgroßschreibung aus der Generalisierung der Großschreibung von Eigennamen entwickelt (vgl. Mentrup 1979: 33, Scheuringer 1996: 20, Bergmann \& Nerius 1998: 871). Sie nehmen im Diskursuniversum eine prominente Stellung ein, da sie für die diskursiven Zusammenhänge des Textes in der Regel eine Schlüsselrolle spielen. In dieser zentralen Rolle liegt eine Ursache, warum Eigennamen die ersten satzinternen Diskursinstanzen waren, die durch initialen Majuskelgebrauch ausgezeichnet wurden. ${ }^{17}$

Einteilige Eigennamen unterscheiden sich bezüglich des Majuskelgebrauchs nicht von anderen Substantivklassen und sind deshalb im Rahmen der Groß- und Kleinschreibung unauffällig. Bei mehrteiligen Eigennamen werden auch attributive Bestandteile großgeschrieben, sodass eine gesonderte Betrachtung und eine Abgrenzung zu anderen Substantivklassen notwendig sind.

Mill (1949: 37) definiert Eigennamen anhand ihres ausschließlich denotativen Charakters. Eigennamen „bedeuten“ nichts, sondern verweisen lediglich auf bestimmte Personen oder Objekte der Welt. Analog definieren Ewald \& Nerius (1990: 97) den Eigennamenbegriff zum Zweck der „Identifizierung bestimmter individueller Objekte und deren Unterscheidung von anderen Objekten der gleichen Art“. Dabei liegt die denotative Funktion deutlich im Fokus, wenngleich die Definition der Tatsache Rechnung trägt, dass mehrteilige Eigen-

16 Der typografische Usus, Text- oder Kapitelanfänge, seltener auch Absätze, durch besondere graphetische Heraushebung des Initialbuchstabens zu kennzeichnen, kann in diesem Sinne als eine Art Superinstantiierung betrachtet werden, die den Abschnittsanfang vom mit ihm zusammenfallenden Ganzsatzbeginn graphetisch abhebt.

17 Weitere Gründe liegen in einer zweiten Funktion der Majuskel innerhalb des deutschen Schriftsystems, auf die in diesem Beitrag nicht eingegangen werden kann, vgl. Anm. 1. 
namen durchaus auch konnotative Bestandteile haben können („Neues Rathaus“, „Sächsische Schweiz“ etc.).

Stetter (1990: 305-307, 1998: 41-43) sieht den entscheidenden Unterschied zwischen Substantiven und Eigennamen darin, dass Substantive zur Kennzeichnung verwendet werden (,X ist ein $\mathrm{Y}^{\prime}$ ), während Eigennamen aus einem Akt der Namensgebung („Dieses ... soll zukünftig ... heißen“) entstünden (Stetter 1998: 43-44). Auf dieser Basis ist sein Gedanke der „ursprünglichen Taufe“ (ebd. 45) zu verstehen, der seiner Auffassung nach konstitutiv für Eigennamen ist und den Vorgang der Namensgebung als zentral in den Vordergrund rückt. Bei der ursprünglichen Taufe können konnotierende Namensbestandteile eine Rolle spielen, bilden aber kein notwendiges Kriterium.

Stetters Ansatz führt $\mathrm{zu}$ einem wesentlich weiteren Eigennamenbegriff als dem von Ewald \& Nerius (1990), da er das Konzept der Individualität weiter fasst.

Das Individuum, das Deutscher Schäferhund heißt, ist die betreffende Hunderasse, Deutscher Meister im Trampolinspringen der gemeinsame Name für die Menge all derjenigen, die jemals diesen Titel gewonnen haben bzw. gewinnen werden (Stetter 1990: 306).

Ganz ähnlich argumentiert Eisenberg (2007: 70):

Das jeweils Identifizierte muss nicht ein einzelnes Ding im Sinne eines einzelnen Individuums sein, sondern kann selbst durchaus aus vielen Einzelwesen bestehen. So identifiziert man mit Lehmann eine Familie in einer Menge von Familien und mit Schwalbenschwanz eine Schmetterlingsart innerhalb der Menge von Arten, die zu den Tagfaltern gehören.

So plausibel diese Ausweitung des Eigennamenbegriffs von Individuen auf individuelle Klassen ist, verwischt sie die Grenze zwischen Eigennamen und Substantiven. Mithilfe einer zu Eisenberg analogen Argumentation ließe sich praktisch jedes Appellativum als Eigenname betrachten.

Die amtlichen Regelungen sind dem engen Eigennamenbegriff gefolgt ( $§ 59$ AR) und trennen zwischen Eigennamen und „festen Verbindungen“ (AR 2.4, §§ 63, 64). Diese Abgrenzung kann das Grundproblem jedoch nicht lösen, sondern verschleiert lediglich die funktionale Ähnlichkeit beider Gruppen. Darüber hinaus verlagert sie das Definitionsproblem zugunsten eines hinreichend klar umrissenen Eigennamenbegriffs auf eine unzureichende Definition der „festen Verbindungen“, deren Extensionsbestimmung auf das Wörterverzeichnis verlagert wird.

Die Ursache für die Ausweitung des Eigennamenkonzeptes auf Objektklassen liegt im Bedürfnis der Sprachgemeinschaft zur Bildung komplexer, auf spezielle Sachverhalte bezogener begrifflicher Terme, die häufig aus AdjektivNomen-Verbindungen bestehen und durch Majuskelschreibung gekennzeich- 
net werden. ${ }^{18}$ Wie mehrteilige Eigennamen verfügen diese Verbindungen über spezifische grammatische Eigenschaften. Beispielsweise erlauben beide keine Attribuierungen zwischen adjektivischem und nominalem Bestandteil. Dieses Verhalten entspricht dem anderer Diskursinstanzen, sofern die Gesamtverbindung - und nicht das Nomen allein - als Diskursinstanz betrachtet wird.

(17) das bekannte Neue Rathaus; ${ }^{*}$ das Neue bekannte Rathaus

(18) das begehrte Gelbe Trikot; * das Gelbe begehrte Trikot

Sowohl für Eigennamen als auch für „feste Verbindungen“ gilt darüber hinaus, dass sie nicht notwendig dem Kompositionalitätsprinzip folgen. So wie das Neue Rathaus nicht neu sein muss, ist das entscheidende Charakteristikum des Gelben Trikots nicht seine Farbe, sondern seine Bedeutung im Radsport. Der Begriff könnte bestehen bleiben, selbst wenn sich die tatsächliche Farbe längst geändert hätte. Die denotative Funktion dominiert die konnotative.

Die textpragmatische Gemeinsamkeit von mehrteiligen Eigennamen und „festen Verbindungen“ liegt darin, dass das vorangestellte Adjektiv nicht als Attribut verstanden werden soll, sondern integraler Bestandteil des Terms ist. Daraus erklären sich sowohl die Besonderheiten bezüglich des Kompositionalitätsprinzips als auch die der Attribuierbarkeit des Gesamtkomplexes. Sie sind das Resultat von Wortbildungsmustern, die nicht nur morphologische, sondern auch syntaktische Bildungsprozesse einbeziehen (vgl. Fuhrhop 2008: 223 f). Für den Großteil ist außerdem ein Akt der bewussten Namensgebung und damit der ursprünglichen Taufe i.S. Stetters konstitutiv, insbesondere innerhalb von Fachsprachen. Da allerdings der weite Eigennamenbegriff Abgrenzungsprobleme $\mathrm{zu}$ anderen Substantivklassen mit sich bringt, werde ich in der Folge von syntaktisch komplexen Termen sprechen, unter denen Eigennamen i. e. S. eine Unterkategorie bilden.

Im Diskursuniversum bilden syntaktisch komplexe Terme nur als Gesamtheit eine Diskursinstanz, was durch Großschreibung des ersten Bestandteils zum Ausdruck kommt. Die zusätzliche Majuskel des nominalen Bestandteils ist eine Besonderheit, die aus den syntaktischen Eigenschaften des Terms resultiert, welche ihrerseits textpragmatische Funktionen erfüllen. Sie resultiert aus dem Dilemma

18 Nerius (2000: 359 f) veröffentlicht eine Liste von Zeitungsbelegen mit Verstößen gegen die damals gültige Norm (Schwarzes Loch, Gelbes Trikot, Große Koalition etc.), die dieses terminologische Bedürfnis auch und gerade bei professionellen Schreibern belegt. 
des Auseinandertretens von Begriffsbeginn und syntaktischem Kern ${ }^{19}$ und verursacht spezifische Erwerbsschwierigkeiten, die sich empirisch in normwidrigen Schreibungen wie Rotesmeer und Sächsischeschweiz niederschlagen. ${ }^{20}$ Sie belegen, dass bereits minder erfahrene Schreibende ein differenziertes Wissen über die äußeren Grenzen von Diskursinstanzen besitzen, die sie in Analogie zu anderen Diskursinstanzen spatienlos und durch genau eine Majuskel kennzeichnen.

$\mathrm{Ob}$ und inwiefern es sich bei einer textlichen Entität um einen syntaktisch komplexen Term handelt, ergibt sich aus dem thematischen Zusammenhang und ist damit nur im Bezug auf den Text zu entscheiden. Während die Verwendung des Gelben Trikots als Begriff Texten des Themas „Profiradsport“ vorbehalten bleibt, können Bekleidungsfachgeschäfte nach wie vor nur gelbe Trikots verkaufen. Der Diskurs und nicht das Lexikon bildet die Ebene, auf der eine sinnvolle Entscheidung über den Majuskelgebrauch getroffen werden kann.

Aus textpragmatischer Sicht erweist sich das Problem von Eigennamen und festen Verbindungen sowohl in begrifflicher Hinsicht, als auch im Zusammenhang der Groß- und Kleinschreibung als innerhalb des Gesamtmodells lösbar. Die Verwendung der Majuskel bei Adjektiv-Nomen-Verbindungen lässt sich als Hinweis an den Leser interpretieren, das Adjektiv nicht als Attribut misszuverstehen und demnach den Gesamtkomplex und nicht nur das Nomen als Diskursinstanz zu betrachten.

19 Syntaktisch komplexe Diskursinstanzen zeigen eine charakteristische Divergenz zwischen dem graphemischen Anfang der Instanz und dem Anfang ihres syntaktischen Kopfes, die sich aus der Systematik der deutschen Getrennt- und Zusammenschreibung ergibt (vgl. Fuhrhop 2009: 56) und bei syntaktisch einfachen Instanzen nicht beobachtbar ist, da der Anfang der Instanz und der des syntaktischen Kopfes graphemisch zusammenfallen. Die Hervorhebung des syntaktischen Kopfes durch Majuskelgebrauch ist diskursiv insofern von Bedeutung, als der Kopf die grammatikalischen Merkmale spezifiziert, die zur anaphorischen Wiederaufnahme der Diskursinstanz benötigt werden. Die volle diskursive Funktionsfähigkeit bedarf daher nicht nur der Spezifizierung des Anfangs der Instanz, sondern auch ihres syntaktischen Kopfes. Jene sichert die intendierte Interpretation als syntaktisch komplexe Diskursinstanz, diese sichert die Funktionalität der Diskursstruktur. Aus analogen Gründen werden auch Anfang und syntaktischer Kopf von Durchkupplungen großgeschrieben.

20 Beide Schreibungen entstammen einem orthografischen Diktatkorpus, das derzeit empirisch ausgewertet wird, vgl. Anm. 1, und traten bei Schülerinnen und Schülern der 7. und 9. Jahrgangsstufe mit einer Häufigkeit von ca. 11 \% (Rotesmeer) bzw. ca. 5 \% (Sächsischeschweiz) auf. 


\section{4 Überschriften und Werktitel}

Im Gegensatz zu bisher untersuchten Textbestandteilen sind Überschriften und Werktitel nicht dem diskontinuierlichen Textmodus zugeordnet und enden daher mit einer Absatzmarkierung (vgl. Bredel 2011: 32-33). Überschriften und Werktitel bilden in der Regel rein thematische Textbestandteile und übernehmen darüber hinaus gliedernde Funktionen. Die Operationalisierungskriterien der Attribuierung und anaphorischen Wiederaufnahme lassen sich nicht anwenden. Dennoch können Überschriften und Werktitel in das Modell der diskursiven Instantiierung integriert werden.

Es ist wiederholt bemerkt worden, dass Überschriften und Werktitel wesentliche funktionale Ähnlichkeiten mit Eigennamen haben. So weisen bereits Ewald \& Nerius (1990: 24) auf die Nähe zwischen Überschriften und Eigennamen hin, betonen zuletzt allerdings die Unterschiede beider Kategorien. Im Gegensatz zu Eigennamen dienten Überschriften und Werktitel v. a. der „Hervorhebung bestimmter inhaltlich-thematischer Aspekte“. „Nicht der Bezug zur lexikalischen Ebene wie bei den Eigennamen steht im Vordergrund, sondern der Bezug zur Textebene“ (Ewald \& Nerius 1990: 104). Diese Auffassung vertritt auch Gallmann (1997: 216), der Buchtitel ausdrücklich näher an Phänomene der Großschreibung am Satzanfang als an Eigennamen heranrückt (vgl. außerdem Augst \& Dehn 2007: 154 f).

Diese Charakterisierung von Überschriften und Werktiteln impliziert die Annahme, dass Überschriften und Werktitel primär konnotative, und höchstens sekundär denotative Funktionen erfüllen. Im folgenden Abschnitt wird dieser Auffassung widersprochen und stattdessen die vorrangig denotative Bedeutung von Überschriften und Werktiteln herausgearbeitet. Davon leitet sich in der Tat eine große funktionale Ähnlichkeit zu Eigennamen ab, aus der sich ihre Großschreibung nach dem Modell der diskursiven Instantiierung ergibt. ${ }^{21}$

Die „Hervorhebung bestimmter inhaltlich-thematischer Aspekte“, die Ewald \& Nerius (1990: 104) als zentral herausstellen, kann nicht als notwendige Bedingung für Überschriften und Werktitel gelten. Das ist bei Werktiteln bereits daraus zu entnehmen, das auch nicht-sprachliche Werke, bspw. der bildenden Kunst oder der Musik, Titel besitzen, die Texttiteln funktional entsprechen. Darüber hinaus finden sich Überschriften, die keine konnotative, sondern lediglich gliedernde Funktionen erfüllen (Kapitel 1, XI, Fünfte Vigilie, etc.), ja sogar solche, die eine inhaltlich-thematische Bezugsmöglichkeit zum Text geradezu programma-

21 Damit soll nicht behauptet werden, dass Überschriften und Werktitel Eigennamen sind. Sie sind diesen aber funktional so ähnlich, dass sich ihr gleichartiges Verhalten bezüglich des Majuskelgebrauchs folgerichtig ableiten lässt. 
tisch verweigern. ${ }^{22}$ Der konnotative Charakter solcher Beispiele ist mindestens problematisch, während der denotative außer Frage steht.

Wichtiger als diese Befunde, die sich unter Aufwendung einiges Scharfsinns möglicherweise in eine konnotative Sichtweise integrieren ließen, ist, dass sich die denotative Funktion immer dann als notwendige Bedingung herausstellen lässt, wenn explizite Intertextualität hergestellt werden soll, d. h. wenn ein Text oder Textabschnitt selbst zum Thema des Diskurses wird. Überschriften und Werktitel verhalten sich in diesen Fällen wie komplexe Eigennamen und unterliegen dann folgerichtig dem kontinuierlichen, statt dem diskontinuierlichen Textmodus. Dieser eigennamenäquivalente Gebrauch von Überschriften und Werktiteln ist nicht der Ausnahme-, sondern der Regelfall. Es gibt nur eine andersartige Verwendung, nämlich die ursprüngliche am Beginn des entsprechenden Textes, die sich ihrerseits unschwer mit Stetters Konzept der ursprünglichen Taufe verbindet.

Zur Herstellung expliziter Intertextualität benötigen Texte bzw. Textabschnitte einen Namen und als solcher fungieren Titel und Überschriften. Dass die denotative Funktion dabei im Vordergrund steht, zeigt sich daran, dass Textteile zwar ohne Überschrift, aber nicht ohne Namen auskommen, sofern sie am intertextuellen Kosmos beteiligt werden sollen. Für Texte und Textbestandteile ohne Überschrift haben sich deshalb verschiedene Praktiken der Denotation eingebürgert, so etwa die Verwendung des Textanfangs (Vater unser, Quempas, Wenn nicht mehr Zahlen und Figuren) ${ }^{23}$ oder die Nutzung der gliedernden Kapitel- und Abschnittsnummerierungen. Mitunter finden sich weitere Phänomene, die sonst nur für Eigennamen typisch sind, so etwa die Verwendung von „Spitznamen“ (Goethes Werther, die Principia Newtons etc.). Es wäre nicht angemessen, darin die „Hervorhebung bestimmter inhaltlich-thematischer Aspekte“ (s. o.) zu sehen. Mit Spitznamen wird die konnotative Funktion der Titel rigoros beschnitten, während die denotative unverändert aufrecht erhalten bleibt.

Aus der Darstellung ergibt sich die lediglich sekundär konnotative Funktion von Überschriften und Werktiteln, die im Diskurs in erster Linie der Bezeichnung bzw. Identifizierung bestimmter individueller textlicher Objekte dienen. Diese Identifizierung geschieht zum Zweck der intertextuellen Bezugnahme und geht

22 Beispielhaft dafür stehen Werke des absurden Theaters wie etwa Ionescos Drama Die kahle Sängerin, dessen Titel aufgrund eines Versprechers entstand. Der Titel wurde damit bewusst als Resultat eines Zufalles bzw. Missverständnisses gewählt und versagte sich damit jeder (direkten) Konnotation. Nicht zu verkennen ist gleichwohl, dass sich ebendiese Verweigerung in indirekter Weise dennoch als Hinweis auf den Inhalt des Stückes verstehen lässt.

23 Dass dabei die Anfänge des Textes, teils ohne Rücksicht auf Wort- oder Phrasengrenzen, terminologisch instrumentalisiert werden, korrespondiert mit der Initialisierungsfunktion des Majuskelgebrauchs. 
als solche auch mit anaphorischen Verweisen und Attribuierungen einher. Überschriften und Werktitel lassen sich damit in das Instanzenmodell der Majuskelgebrauchs integrieren. In ihrer ursprünglichen Verwendung - als Beginn des Textes oder Textabschnitts - bilden sie die maximale Instanz des Textes, in allen anderen Verwendungsweisen einen den Eigennamen funktional eng verwandten komplexen Term.

\subsection{Pronomina und Höflichkeitsanreden}

Im Gegensatz zu anderen Pronomina gehören Anreden teilweise zum Gegenstand des amtlich geregelten Majuskelgebrauchs. In der zweiten Person werden Höflichkeitsanreden obligatorisch, informelle Anreden optional durch Großschreibung initialisiert. Auch historisch bilden sie die einzige nennenswerte Häufung pronominaler Großschreibungen (vgl. Bergmann \& Nerius 1998: 731). Nachdem die Rechtschreibreform von 1996 die Großschreibung ausschließlich auf die Höflichkeitsanrede beschränkt hatte, wurde diese restriktive Auslegung aufgrund der geringen Akzeptanz der Sprachgemeinschaft 2006 wieder liberalisiert (vgl. § 66 E).

Im Gegensatz zur 3. Person erfüllen Pronomina der 1. und 2. Person nicht anaphorische, sondern deiktische Funktionen, beinhalten also keinen Verweis auf eine Diskursinstanz, sondern können selbst als solche aufgefasst werden. Sofern Textemittent und Textadressat überhaupt explizit in die propositionale Struktur des Textes eingebunden sind, nehmen sie eine eigenständige Rolle im Diskursuniversum ein, ohne der Instantiierung durch eine Nominalgruppe zu bedürfen. Dass für die Großschreibung die deiktische Verwendung und nicht das grammatische Merkmal (1. bzw. 2. Person) ausschlaggebend ist, zeigt die heute veraltete Großschreibung von Anredepronomina in der 3. Person Sg., die in den alten Regeln noch erwähnt wurde („Schweig Er! Höre Sie!“”, vgl. R 122).

Daneben treten bisweilen sprachliche Verwendungsweisen von Pronomina als eigenständige Diskursinstanzen auf, die regelmäßig mit Majuskelgebrauch einhergehen. Sie sind diskursiv stark restringiert, weil der Bezug auf ein definiertes außersprachliches Referenzobjekt nur bedingt gewährleistet werden kann. So werden uneingeführte Pronomina der 3. Pers. Sg. mask. in der Regel auf eine göttliche Instanz bezogen. Majuskelgebrauch kann diese Interpretation auch gegen potenzielle alternative Bezugsmöglichkeiten erzwingen.

(19) Fritz hat den Dackel getreten. Das wird er vor Ihm verantworten müssen.

Außerhalb des Gottesbezuges ist die Verwendung nicht-anaphorischer Pronomina als Diskursinstanz selten, aber belegt. Sie führt etwa zu einer Interpretation als generischer Sexus (20) oder als Bezeichnung von etwas sprachlich Unfassbarem 
(21) und geht in dieser Funktion bisweilen auch mit initialer Majuskel einher, obwohl die amtliche Norm diesen Gebrauch nicht vorsieht.

(20) Ruhig bleiben, wenn Sie Auto fährt. ${ }^{24}$

(21) Und dann bricht Es wie die Pest über die Stadt Derry herein. ${ }^{25}$

Die Beispiele belegen, dass auch Pronomina zu Großschreibung neigen, sofern sie nicht anaphorisch auf eine Diskursinstanz verweisen, sondern selbst eine bilden. Es ist daher weniger die Großschreibung der Höflichkeitsanrede, als vielmehr die Kleinschreibung von $d u$ und $i h r$ sowie der Pronomina der 1. Person erklärungsbedürftig. Sie ist möglicherweise das Resultat einer schriftsprachlichen Dilemmasituation, deiktische Pronomina entweder in Analogie zu anderen pronominalen Ausdrücken klein oder in Analogie zu anderen Diskursinstanzen großzuschreiben. Da Emittent und Adressat nur in bestimmten Textsorten als explizite textinterne Instanzen auftreten, erfolgt Großschreibung nur dann, wenn sie durch zusätzliche diskursive Kriterien motivierbar ist, etwa durch besondere ehrerbietende Auszeichnung, die historisch belegt ist und neben der Höflichkeitsanrede auch die Großschreibung im Pluralis Majestatis verursacht.

Bei der informellen Anrede scheint Großschreibung und damit die Auszeichnung als Diskursinstanz je nach Textsorte unterschiedlich angemessen. So war die Großschreibung von $d u$ und ihr nach der alten Rechtschreibung textsortenabhängig geregelt. ${ }^{26}$ Die Gemeinsamkeit derjenigen Textsorten, für die Großschreibung der Anreden vorgesehen war, liegt neben einem gewissen Grad an Ehrerbietung vor allem darin, dass der Adressat in der Regel genau bestimmbar ist und oft in einer persönlichen Beziehung zum Emittenten steht. Beide sind damit in besonderer Weise in die Diskursstruktur eingebunden und lassen sich stärker als in anderen Textsorten als eigenständige, identifizierbare und identifikationsbedürftige Instan-

24 Auszug der Titelseite von Augustin, Eduard et al. (2009). Ein Mann ein Buch. 1. Aufl. München: Goldmann.

25 Auszug aus dem Klappentext von King, Stephen (2009). Es. Neuausgabe. Berlin: Ullstein.

26 Großschreibung galt in „Briefen, feierlichen Aufrufen und Erlassen, Grabinschriften, Widmungen, Mitteilungen des Lehrers an einen Schüler unter Schularbeiten, auf Fragebogen, bei schriftlichen Prüfungsaufgaben usw.“ (R 71), Kleinschreibung hingegen bei der „Wiedergabe von Reden, Dialogen u. ä., in Protokollen, Prospekten, Lehrbüchern“ (R 71.1). Bei „Fragenbogen“ und „Prüfungsaufgaben“ ist der Kontext der informellen Anrede zu berücksichtigen, um der vorgeschlagenen Deutung zu entsprechen. 
zen des Textes auffassen. ${ }^{27}$ Die prototypische Textsorte, in der Emittent wie Rezipient als Diskursinstanzen auftreten, ist der Brief, der mit § $66 \mathrm{E}$ AR die einzige weiterhin amtlich zugelassene Textsorte für die Großschreibung von $d u / i h r$ ist. Die Kleinschreibung der 1. Person außerhalb des Pluralis Majestatis ist neben dem Wegfall der Ehrerbietung möglicherweise über die Wirkung von Bescheidenheitstopoi erklärbar. Eine genauere Rechtfertigung bedarf spezialisierter Untersuchungen, die an dieser Stelle nicht geleistet werden können. Die Ausführungen zeigen jedoch, dass die Ausweitung des Majuskelgebrauchs auf Anredepronomina die textpragmatische Perspektive stützt, obschon die Markierung deiktischer Diskursinstanzen nicht konsequent erfolgt.

\section{Ausblick}

Über textpragmatische Gesichtspunkte lassen sich zentrale Verwendungsweisen der Majuskel unter ein gemeinsames theoretisches Modell subsumieren, das die etablierten grammatisch-formalen Ansätze textlich-funktional ergänzt. Das Modell ist in der Lage, satzinternen, satzinitialen sowie textinitialen Majuskelgebrauch in ein rekursiv-hierarchisches Modell zu integrieren, und erklärt damit ihre graphemische Verwandtschaft. Darüber hinaus kann es begründen, warum etablierte Proben, namentlich die Attribuierungsprobe, für die Groß- und Kleinschreibung relevant sind.

Ein wichtiges Zeichen für die Adäquatheit des textpragmatischen Ansatzes ist seine Vereinbarkeit mit etablierten orthografischen Theorien. Jedes eindeutige Substantiv bzw. jede attribuierbare Nominalgruppe ist auch eine eindeutige Diskursinstanz. Darüber hinaus kann das textpragmatische Modell eine Reihe von Phänomenen begründet integrieren, die nach dem wortkategorialen bzw. syntaktischen Ansatz nur beschrieben und in die AR über die Annahme von Sonder- und Ausnahmeregeln einbezogen werden konnten. Deshalb erscheint es berechtigt, den textpragmatischen Ansatz als erklärungsadäquate Funktion des deutschen Majuskelgebrauchs zu betrachten.

Die textpragmatische Deutung des deutschen Majuskelgebrauchs ist nicht nur eine konfirmatorische Bestätigung des gegenwärtigen (eher noch: des vor der

27 Für diese Deutung spricht auch, dass „du“ in Sprichwörtern und Redewendungen stets kleingeschrieben wird („Hast du keinen, fang dir einen“), selbst wenn eine solche in Briefen mit großgeschriebenem „du“ auftritt. Der Befund, auf den mich Norbert Fries hingewiesen hat, zeigt, dass der Charakter von „du“ als Diskursinstanz nicht von dem Wort selbst, sondern von seinem Bezug abhängt. Das unpersönliche, nicht auf den Adressaten bezogene „du“ gilt dabei nicht als Diskursinstanz. 
Rechtschreibreform bestehenden) Majuskelgebrauchs, sondern bildet darüber hinaus einen Ausgangspunkt für die Beurteilung normativer Eingriffe in das Schriftsystem. Sie eröffnet ferner neue Möglichkeiten, die Hypothese der leseerleichternden Funktion der Groß- und Kleinschreibung $\mathrm{zu}$ untersuchen, und bietet auch didaktisch relevante Ansatzpunkte, auf die im Rahmen dieser Arbeit nicht eingegangen werden konnte. Ebenfalls nur angedeutet wurden weitere Phänomene des deutschen Majuskelgebrauchs, so etwa die mehrfache Anwendung der Majuskel in Durchkupplungen und mehrteiligen Eigennamen sowie die Schreibung stereotyper Phrasen und fester Gefüge. Ihre ausführliche Diskussion bleibt folgenden Beiträgen vorbehalten.

\section{Literatur}

Adelung, Johann Christoph. 1978 [1788]. Vollständige Anweisung zur Deutschen Orthographie nebst einem kleinen Wörterbuche für die Aussprache, Orthographie, Biegung und Ableitung. Hildesheim: Olms.

Augst, Gerhard, Karl Blüml \& Peter Gallmann (eds.). 2007. Die Arbeit der Zwischenstaatlichen Kommission für deutsche Rechtschreibung von 1997 bis 2004. Hildesheim, Zürich, New York: Olms.

Augst, Gerhard \& Mechthild Dehn. 2007. Rechtschreibung und Rechtschreibunterricht. Eine Einführung für Studierende und Lehrende aller Schulformen. 3. Aufl. Stuttgart, Leipzig.

Bergmann, Rolf \& Dieter Nerius. 1998. Die Entwicklung der Großschreibung im Deutschen von 1500 bis 1700. Heidelberg: Winter.

Bock, Michael, Gerhard Augst \& Immo Wegner. 1985. Groß oder Klein? Zur Funktion des Wortanfangs für den gegenwärtigen Leser. Zeitschrift für Entwicklungspsychologie und pädagogische Psychologie 17(3). 191-209.

Bock, Michael, Klaus Hagenschneider \& Alfred Schweer. 1989. Zur Funktion der Groß- und Kleinschreibung beim Lesen deutscher, englischer und niederländischer Texte. In Eisenberg, Peter \& Hartmut Günther (eds.), Schriftsystem und Orthographie. (Reihe Germanistische Linguistik 97), 23-55. Tübingen: Niemeyer.

Bock, Michael. 1989. Lesen in Abhängigkeit von der Groß- und Kleinschreibung. Sprache und Kognition 8(3). 133-151.

Bredel, Ursula, \& Hartmut Günther. 2000. Quer über das Feld das Kopfadjunkt. Bemerkungen zu Peter Gallmanns Aufsatz Wortbegriff und Nomen-Verb-Verbindungen. Zeitschrift für Sprachwissenschaft 19. 103-110.

Bredel, Ursula. 2010. Die satzinterne Großschreibung. System und Erwerb. In Bredel Ursula, Astrid Müller \& Gabriele Hinney (eds.), Schriftsystem und Schrifterwerb: linguistisch didaktisch - empirisch, 217-234. Berlin, New York: De Gruyter.

Bredel, Ursula. 2011. Interpunktion. Heidelberg: Winter.

Eisenberg, Peter. 2007. Grundregeln der deutschen Rechtschreibung. Die deutsche Orthografie auf einen Blick. Gütersloh, München: Wissen Media.

Ewald, Petra \& Dieter Nerius. 1990. Die Groß- und Kleinschreibung im Deutschen. Die geltende Regelung, Problemfälle und Schwierigkeiten, 2. Aufl. Leipzig: Bibliographisches Institut. 
Fuhrhop, Nanna. 2007. Zwischen Wort und Syntagma: Zur grammatischen Fundierung der Getrennt- und Zusammenschreibung. Tübingen: M. Niemeyer.

Fuhrhop, Nanna. 2008. Das graphemische Wort im Deutschen. Eine erste Annäherung. In Zeitschrift für Sprachwissenschaft 27 (2), S. 189-228.

Fuhrhop, Nanna. 2009. Orthografie. 3. Aufl. Heidelberg: Winter (Kurze Einführungen in die germanistische Linguistik, 1).

Gallmann, Peter. 1997. Konzepte der Nominalität. In Augst, Gerhard (ed.), Zur Neuregelung der deutschen Orthographie. Begründung und Kritik (Reihe Germanistische Linguistik 179), 209-241. Tübingen: Niemeyer.

Gallmann, Peter \& Horst Sitta. 2007. Die Neuregelung der deutschen Rechtschreibung. Bern: Paritätische Berufsbildungsstelle für visuelle Kommunikation.

Glinz, Hans. 1984. Sätze: Einheiten für das Hören/Lesen - Einheiten des grammatischen Baus. Praxis Deutsch 68. 43-47.

Gottsched, Johann Christoph. 1970 [1749]. Vollständigere und Neuerläuterte Deutsche Sprachkunst, In Rössing-Hager, Monika (ed.), Deutsche Grammatiken des 16. bis 18. Jahrhunderts (Documenta Linguistica. Quellen zur Geschichte der deutschen Sprache des 15.-20. Jahrhunderts, Reihe V). Hildesheim: Olms.

Grüter, Majana. 2009. Optimalitätstheoretische Modellierung der Groß- und Kleinschreibung: eine Beispielanalyse englischer Texte um 1730. Zeitschrift für Sprachwissenschaft 28(2). 203-230.

Günther, Hartmut \& Désirée-Kathrin Gaebert. 2011. Das System der Groß- und Kleinschreibung. In: Bredel, Ursula \& Tilo Reißig (eds.), Weiterführender Orthographieerwerb, 96-106. Baltmannsweiler: Schneider.

Günther, Hartmut \& Ellen Nünke. 2005. Warum das Kleine groß geschrieben wird, wie man das lernt und wie man das lehrt. In Günther, Hartmut \& Michael Becker-Mrotzek, Kölner Beiträge zur Sprachdidaktik 1. http://www.koebes.uni-koeln.de/guenther_nuenke.pdf (06. 03. 2012).

Ickler, Theodor. 1999. Kritischer Kommentar zur „Neuregelung der deutschen Rechtschreibung“ mit einem Anhang zur „Mannheimer Anhörung“, 2. Aufl. Erlangen \& Jena: Palm und Enke.

Kamp, Hans \& Uwe Reyle. 1993. From Discourse to logic. Introduction for Modeltheoretic Semantics of Natural Language, Formal Logic and Discourse Representation Theory. Dordrecht, Boston, London: Kluwer Adacemic Publishers.

Karttunen, Lauri. 1976. Discourse referents. In McCawley, James David (ed.), Syntax and Semantics 7: Notes from the Linguistic Underground, 363-385. New York: Academic Press.

Kluge, Wolfhard. 1985. Zur Großschreibung der Substantive. Einige kritische Anmerkungen linguistischer und methodischer Art. In Hoberg, Rudolf (ed.), Rechtschreibung im Beruf, (Reihe Germanistische Linguistik 56), 63-78. Tübingen: Niemeyer.

Maas, Utz. 1992. Grundzüge der deutschen Orthographie. 2. korrigierte und erw. Aufl. Tübingen: Niemeyer.

Maas, Utz. 2007. Die Grammatikalisierung der satzinternen Großschreibung. In Redder, Angelika. (ed.) Diskurse und Texte. Festschrift für Konrad Ehrlich zum 65. Geburtstag. 385-399. Tübingen: Stauffenburg.

Mentrup, Wolfgang. 1979. Die Groß- und Kleinschreibung im Deutschen und ihre Regeln. Historische Entwicklung und Vorschlag zur Neuregelung, Tübingen: Niemeyer.

Mentrup, Wolfgang. 1980. Materialien zur historischen entwicklung der gross- und kleinschreibung. Tübingen: Niemeyer.

Mill, John Stuart. 2002 [1949]. A System of Logic. Ratiocinative and inductive: being a connected view of the priciples of evidence and the methods of scientific investigations. Honolulu: University Press of the Pacific. 
Munske, Horst Haider. 2005. Die angebliche Rechtschreibreform. St. Goar: Leibnitz.

Nerius, Dieter. 2000. Zur Groß- und Kleinschreibung fester nominaler Wortgruppen in der gegenwärtigen und künftigen deutschen Orthographie. In: Ewald, Petra \& Bernd Skibitzki (eds.), Beiträge zur deutschen Orthographie. Herausgegeben anlässlich des 65. Geburtstages von Dieter Nerius, 359-367. Frankfurt/M. u. a.: Lang.

Noack, Christina. 2006. „Aber Wie-Wörter schreibt man doch klein“. Groß- und Kleinschreibung: Warum der Unterricht oft Zweifel produziert - und wie sich das ändern lässt. Praxis Deutsch 198. 36-43.

Nünke, Ellen \& Christiane Wilhelmus. 2001. Stufenwörter in Treppengedichten. Ein alternativer Ansatz zur Groß- und Kleinschreibung. Praxis Deutsch, 28(170). 20-23.

Ossner, Jakob. 2010. Orthographie. System und Didaktik. Paderborn: Schöningh.

Primus, Beatrice. 2010. Strukturelle Grundlagen des deutschen Schriftsystems. In Bredel, Ursula, Astrid Müller \& Gabriele Hinney (eds.), Schriftsystem und Schrifterwerb: linguistisch - didaktisch - empirisch, 9-45. Berlin, New York: De Gruyter.

Röber-Siekmeyer, Christa. 1999. Ein anderer Weg zur Groß- und Kleinschreibung. Leipzig, Stuttgart, Düsseldorf: Klett.

Scheele, Veronika. 2006. Entwicklung fortgeschrittener Rechtschreibfertigkeiten. Ein Beitrag zum Erwerb der „orthographischen“ Strategie, Hannover 2005 Dissertation. Frankfurt/M: Lang.

Scheuringer, Hermann. 1996. Geschichte der deutschen Rechtschreibung. Ein Überblick. Mit einer Einführung zur Neuregelung ab 1998, Wien: Ed. Praesens.

Schwarz, Monika \& Jeannette Chur. 2004. Semantik. Ein Arbeitsbuch. 4. Aufl. Tübingen: Narr.

Stein, Stephan. 1999. Majuskeln im WortInnern. Ein neuer graphostilistischer Trend für die Schreibung von Komposita in der Werbesprache. Muttersprache 109. 261-278.

Stetter, Christian. Gibt es ein graphematisches Teilsystem der Sprache? Die Großschreibung im Deutschen. In Eisenberg, Peter \& Hartmut Günther (eds.), Schriftsystem und Orthographie. (Reihe Germanistische Linguistik 97), 297-320. Tübingen: Niemeyer. 